\title{
A double mirror W/C multilayer monochromator for radiation biology applications
}

\author{
J. F. MacKay, D. W. Pearson, B. E. Nelms, P. M. DeLuca, Jr., and M. N. Gould \\ Departments of Medical Physics and Human Oncology, University of Wisconsin, Madison, Wisconsin 53706 \\ M. G. Lagally \\ Departments of Physics and Materials Science, University of Wisconsin, Madison, Wisconsin 53706
}

(Received 17 April 1997; accepted for publication 5 March 1998)

\begin{abstract}
A double-mirror multilayer monochromator was developed for the purpose of irradiating live cell cultures at the Synchrotron Radiation Center, University of Wisconsin-Madison. The monochromator is designed for the soft $\mathrm{x}$-ray region with photon energies between 270 and $2400 \mathrm{eV}$. Multilayer mirrors with 55 bilayers of W/C and a bilayer spacing of $d=3.0 \mathrm{~nm}$ are sputter deposited on Si substrates. By proper masking of the sputtering sources, variation in the bilayer spacing over the area of the mirror is minimized. The uniformity of the bilayer spacing was measured to be $\Delta d / d<1 \%$, over the $75 \mathrm{~mm} \times 25 \mathrm{~mm}$ area of the mirrors. The reflectivity was measured as a function of energy to determine the integrated reflectivity and evaluate the contribution of the specular reflection and higher orders to the monochromatic beam. The use of suitable filters with a $\mathrm{Si}(\mathrm{Li})$ detector allows determination of the spectral output of the monochromator. The output power of the monochromator between 270 and $2400 \mathrm{eV}$ is measured. The resolution of the monochromator is $\Delta \lambda / \lambda=0.04$. Applications of the monochromator to radiation biology are discussed. (C) 1998 American Association of Physicists in Medicine. [S0094-2405(98)02905-8]
\end{abstract}

\section{INTRODUCTION}

Layered synthetic microstructures, also known as multilayer mirrors, have recently shown promise as optical elements in synchrotron radiation beamlines. Multilayer mirrors are used as power filters, polarimeters and monochromators. ${ }^{1-3}$ Several researchers reported development of prototype doublemirror monochromators. ${ }^{4,5}$ More recently Malek reported a $\mathrm{Mo} / \mathrm{C}$ double-mirror monochromator for use in $\mathrm{x}$-ray lithography photoresist studies. ${ }^{6}$ Such monochromators have the advantage that the multilayers may be tailored to a specific application or energy range while retaining the inherent energy bandpass. Although a multilayer monochromator has limited energy resolution, for many applications the resolution is adequate. In particular, the trade off of higher flux for lower resolution is often a considerable benefit.

An important application of soft $\mathrm{x}$ rays is in the area of radiation biology. In several studies, researchers have used $\mathrm{C}$ $K \alpha$ or Al $K \alpha$ radiation to study cell survival as a function of irradiation absorbed dose. ${ }^{7,8}$ These studies were limited to available emission lines that could not be tuned for two wavelengths with equal attenuation in the cell. Synchrotron radiation would seem an obvious choice for variable wavelength studies, yet biological research at the typical synchrotron facility presents a unique set of challenges. Living tissue is incompatible with ultrahigh vacuum (UHV). Most existing synchrotron monochromators are designed to provide excellent energy resolution, with consequent low intensity and with the $x$ rays focused to a small area. This situation is desirable for spectroscopy experiments such as electron photoemission, but for radiation biology, large numbers of living cells must be irradiated uniformly and a small irradiation field is a severe limitation. In addition, much of the interest in biological applications is in an energy range from 200 to
$2000 \mathrm{eV}$ in which most x-ray grating monochromators do not perform well. For radiation biology, the primary considerations are intensity and uniformity of the irradiating beam. The intensity is critical because it determines the time required to achieve a given absorbed dose. The beam must be uniform and physically large enough to ensure that all cells within a culture dish receive the same dose. Radiation biology is just the sort of application that is well suited for a multilayer monochromator.

For our needs, the basic requirements of the monochromator are an output energy range from 270 up to $2000 \mathrm{eV}$, a beam size of at least $25 \mathrm{~mm}$, and an energy variation across the output beam constrained to be less than $1 \%$. W/C multilayers with bilayer spacing of $3.0 \mathrm{~nm}$ were chosen because they cover the desired energy range with acceptable reflectivity. This report discusses the beamline and the development of the required multilayers, as well as techniques to irradiate live cells and measure the dose.

\section{MULTILAYER MIRRORS}

\section{Multilayer growth}

The W/C multilayer mirrors are grown in a dc magnetron sputtering system, with a typical base pressure of $40 \mu \mathrm{Pa}$. During growth, purified Ar is introduced into the system to generate the sputtering plasma. The pressure of the Ar is kept as low as possible $(0.2 \mathrm{~Pa})$ to minimize shadowing, which increases growth roughness that reduces the reflectivity of the multilayer. The configuration of the sputtering system is illustrated in Fig. 1. The sputter sources are $100 \mathrm{~mm}$ in diameter with circular magnets and are placed on opposite sides of the growth chamber. The sputtering targets are highpurity $\mathrm{W}$ and amorphous graphite. As shown in Fig. 1, masks 


\section{Sputtering System}

(Top View)
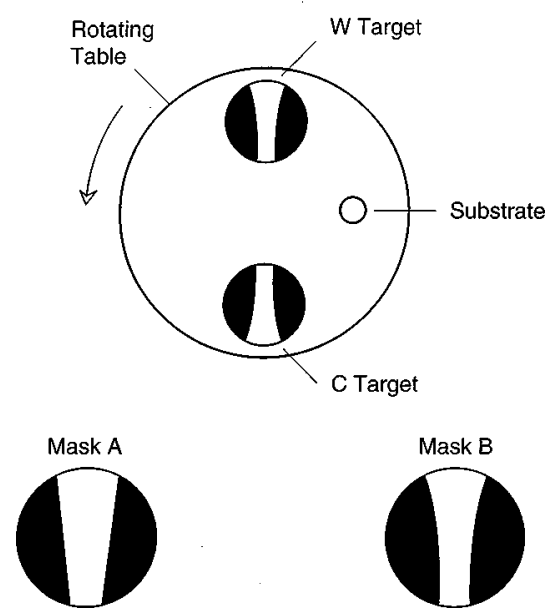

FIG. 1. The sputtering system configuration. The substrates rotate over the masked circular sputtering targets in the sputter up configuration. Mask A compensates only for the nonuniform deposition due to the rotation of the substrate over the target. Mask B compensates for the table rotation and nonuniform deposition due to the circular shape of the targets.

are placed over the targets to control the uniformity of the deposition. Substrates are mounted inside the chamber on a 0.48 -m-diam rotating table driven by an external computer controlled stepping motor. With the substrates face down, alternate layers of $\mathrm{W}$ and $\mathrm{C}$ are deposited as the table rotates. The layer thickness is controlled by varying the speed of rotation over the targets.

\section{Reflectivity measurements}

To evaluate the quality of the multilayers for the monochromator, the peak reflectivity of the multilayers was measured. Figure 2 compares the measured and calculated reflectivity for $d=3.0 \mathrm{~nm}, 55$ bilayer $\mathrm{W} / \mathrm{C}$ multilayers used in the monochromator. Note that the measured absolute peak reflectivity is about $10 \%$ of the incident beam below the $\mathrm{C} K$ edge, then drops dramatically at the edge before rising again above it. This behavior results from photon absorption in the $\mathrm{C}$ layers. At about $1800 \mathrm{eV}$ the reflectivity is reduced because of absorption in the Si substrate below the multilayer film. Over the energy range, the measured reflectivity is about $60 \%$ of the calculated value.

We also measured the energy resolution of the $d=3.0 \mathrm{~nm}$ 55 bilayer multilayers that are used in the monochromator. Resolution is measured by rocking both the multilayer and the detector through the Bragg condition. In this way, the change in reflected intensity for an angular change in $\theta$, with fixed excitation energy, is measured. The change in $\theta$ that results in a decrease in reflected intensity by half gives the full width at half maximum (FWHM) for the multilayer. The measured resolution at $860 \mathrm{eV}$ was 4\% FWHM. The resolution of a given multilayer mirror depends, to some extent, on the number of bilayers. However, the advantage gained by increasing the number of bilayers is limited by the absorption of $x$ rays into the multilayer.

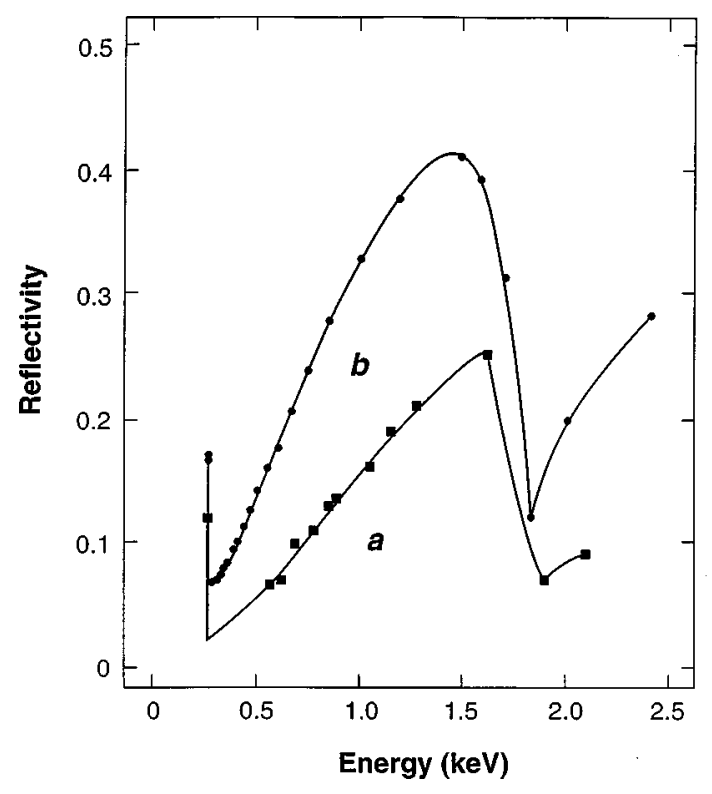

FIG. 2. Absolute reflectivity as a function of energy for the W/C multilayers with 55 bilayers, $d=3.0 \mathrm{~nm}$ used in the monochromator: (a) measured values, (b) calculated values.

\section{Multilayer uniformity}

Both interfacial roughness and layer nonuniformity decrease the reflectivity of a double-mirror monochromator. Nonuniformity can be critical, because a mismatch in the layer spacing of the two mirrors can dramatically reduce the throughput for the pair. For the sputtering configuration in Fig. 1, two sources of nonuniform deposition dominate. The first is due to the rotation of the substrates over the targets. Because the angular velocity is a function of distance from the center of the rotating table, the deposition rate decreases with radial distance. This type of nonuniformity can be compensated using mask "A" shown in Fig. 1." The second cause of nonuniformity is the shape of the sputtering sources. A circular magnetron source generates a ring-shaped sputtering track because more target material is removed where the magnetic field is highest. In our system, the result is a ringshaped growth source about $50 \mathrm{~mm}$ in diameter. The intensity distribution for a ring can be calculated for a fixed height from the target by integrating over the ring, assuming a $1 / r^{2}$ dependence. Figure 3 shows calculated values of the relative deposition rate from a ring-shaped sputtering target that illustrate the problem. The overall effect of this type of nonuniform deposition is a radial variation of the $d$ spacing across the substrate. ${ }^{6}$ The part of the substrate that passes over the center of the target has the largest $d$ spacing, with decreasing values symmetrically on either side of the center. To improve the uniformity of our multilayers, two types of masks were tested. Figure 4 (filled squares) shows $\Delta d / d$ values as a function of position across a $100-\mathrm{mm} \mathrm{W} / \mathrm{C}$ multilayer mirror with mask A over the targets to compensate for the rotation of the substrate. The measurement was done at Aladdin on the Mark II Grasshopper monochromator, ${ }^{10}$ with the multilayer under test held at a fixed angle. The detector was fixed at $2 \theta$, and the output energy of 


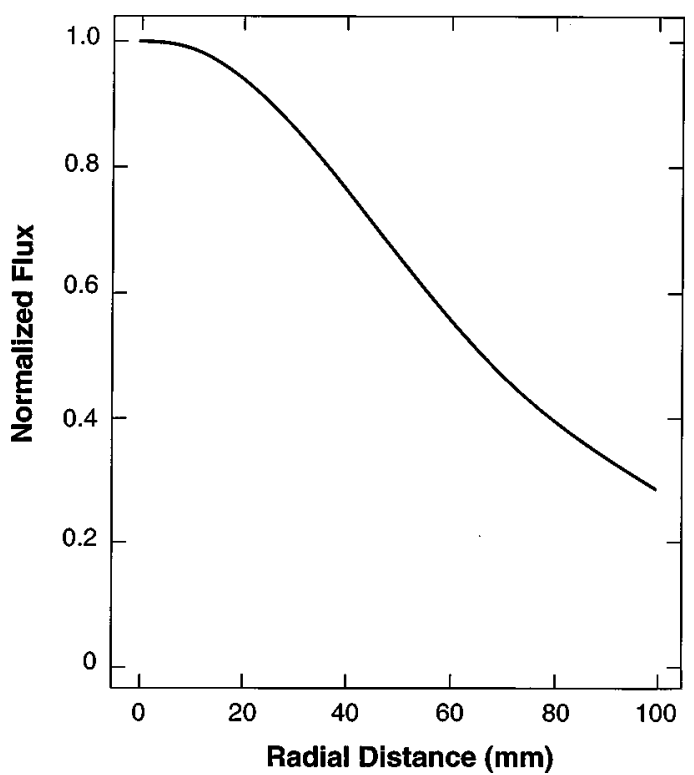

FIG. 3. Calculated deposition flux as a function of radial distance from the center of a 50-mm-diam circular sputtering track. The flux intensity is normalized to the value at the center of the source. The values were calculated assuming a $1 / r^{2}$ dependence in the sputtering flux.

the monochromator was scanned through the Bragg peak. The sample was then translated in increments through the beam, and the energy scans repeated to yield the energy of the Bragg peak for fixed $2 \theta$ along the radial direction of the substrate. The variation in $d$ spacing caused by nonuniform deposition from the sputtering targets is clear, and is in agreement with behavior expected for a ring-shaped source (Fig. 3). Note that $d$ has decreased by $10 \%$ at $25 \mathrm{~mm}$ from the center of the deposition source. By curving the edges of mask A to increase the deposition rate away from the center of the target, one can compensate for the source shape. For simplicity, we chose to use an arc. Figure 4 (filled circles) shows results of a measurement of the variation in $d$ spacing of a W/C multilayer produced using mask $\mathrm{B}$ with an arc radius of $400 \mathrm{~mm}$. Clearly, the center of the sample is more uniform, with a variation of $<2 \%$ over the center $50 \mathrm{~mm}$ of the wafer. From the results of these measurements, it was a simple matter to correct the deposition further. By using an arc radius of $250 \mathrm{~mm}$, the nonuniformity over the center 60 $\mathrm{mm}$ of the wafer was decreased to less than $1 \%$ (results not shown).

\section{Multilayer roughness}

For a multilayer mirror, interfacial roughness reduces the intensity of the Bragg reflection by scattering $\mathrm{x}$ rays away from the specular direction. ${ }^{11}$ Factors that contribute to roughness are the initial roughness of the substrate, interlayer diffusion, sputtering pressure, and the angle of deposition. Ideally, the substrates should be polished to better than $1 \AA$ rms roughness. Interlayer diffusion is a function of the materials chosen for the multilayer, and is therefore more difficult to control. One benefit of masking the sputtering targets is to reduce the amount of low-angle deposition that results

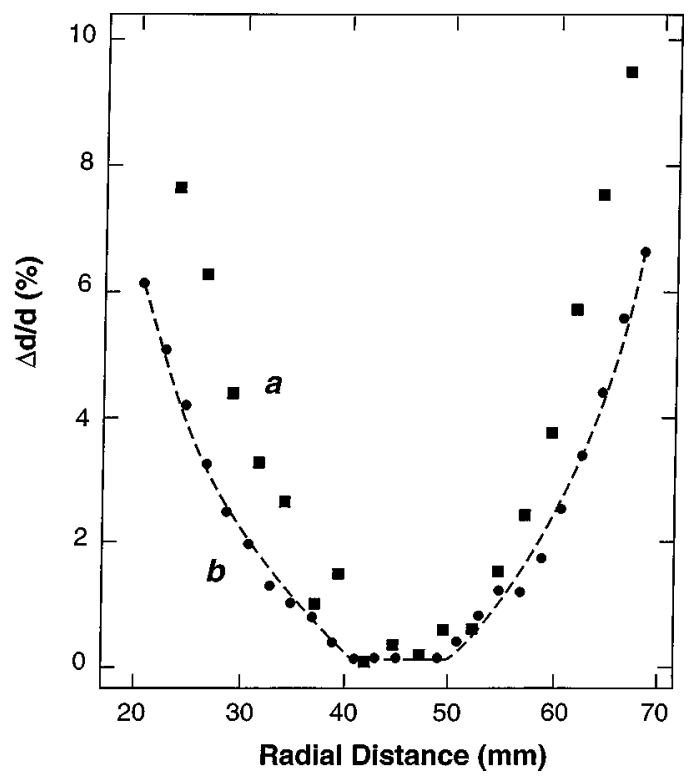

FIG. 4. Measured change in $d$ spacing as a function of radial distance for a W/C multilayer near the center of a $100-\mathrm{mm} \mathrm{Si}$ wafer. The distance is measured from the edge of the wafer. (a) Measured change in the $d$ spacing using mask A in Fig. 1 to compensate for rotation of the substrate over the sputtering target; (b) measured change in the $d$ spacing using mask B in Fig. 1 to compensate for substrate rotation and the shape of the sputtering source. The line is drawn to guide the eye.

in roughness due to shadowing. ${ }^{12}$ The roughness correlated with a Bragg reflection from a multilayer can be measured by performing a rocking curve analysis about any Bragg reflection. ${ }^{11,13}$ The excitation energy is chosen to correspond to a Bragg reflection with the detector fixed at $2 \theta$, where $\theta$ is the Bragg angle. Next the angle of the multilayer under test is varied continuously to scan through the Bragg peak while leaving the detector fixed. The resulting intensity versus angle measurement is the sum of the specular Bragg reflection and a diffusely scattered component. From the diffuse component, one can derive the rms correlated roughness. ${ }^{11,13}$ Figure 5 shows the measured rocking curve for a $d=3.0 \mathrm{~nm}$, 55 bilayer W/C multilayer grown on a 75-mm Si substrate that was used in the monochromator. Note that the rms correlated roughness is about $0.9 \AA$.

\section{Si substrates}

All of the multilayer characterization and development was done using 75 - or 100 -mm-diam $\mathrm{Si}(100)$ wafers. The advantages of this substrate are low cost and availability, and the fact that the polished surface is of very high quality. Unfortunately, Si wafers are not ideal elements in a doublemirror monochromator. The main difficulty is that the wafers are so thin that they cannot be mounted easily without introducing optical distortion. Further distortion is caused by the heat load of synchrotron light and the strain induced by the multilayer. The heat induced distortion is differential as the first mirror absorbs the majority of the heat load. We were able to overcome this problem for development purposes by bonding the $\mathrm{Si}$ wafers (precoated with a multilayer) to polished Mo mirrors. The bonding agent was a low-viscosity 


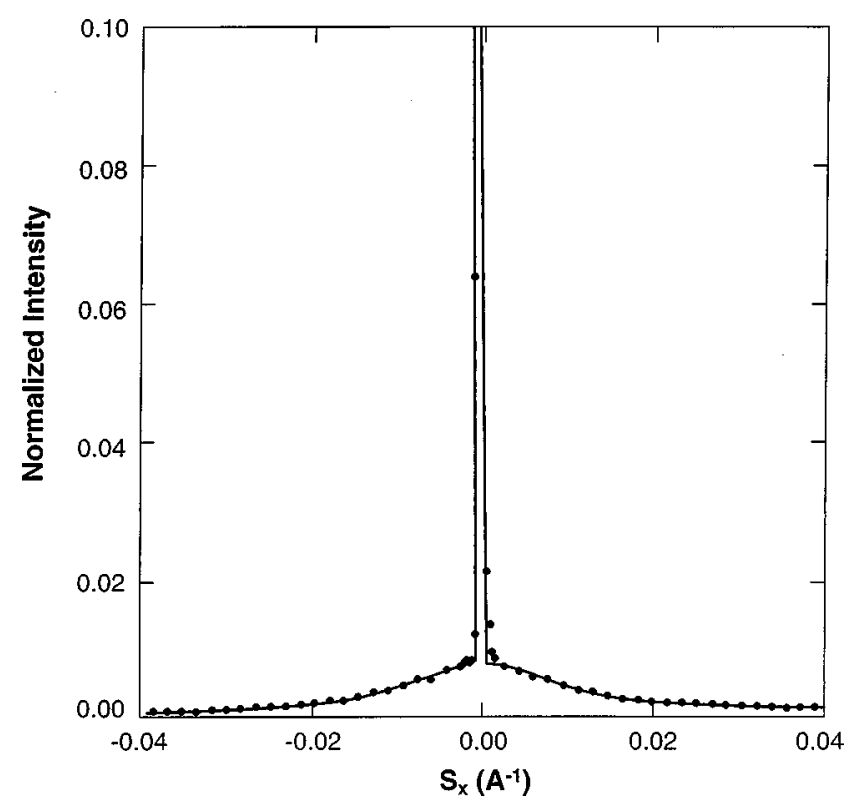

FIG. 5. Scattered photon intensity for a rocking scan of the W/C multilayer with 55 bilayers and $d=30 \mathrm{~nm}$. The intense $n=1$ Bragg peak and the diffuse scattered background are clearly observed. These measurements yield a correlated roughness value of $0.9-\AA ̊$ rms.

epoxy used in x-ray lithography. Other bonding agents that we tried tended to strain and distort the wafers. We find that a set of mirrors prepared in this manner can be used for many years (30 h per week) before the $\mathrm{Si}$ substrate begins to delaminate. Improved mirrors will use polished Si blocks $150 \mathrm{~mm} \times 30 \mathrm{~mm} \times 10 \mathrm{~mm}$ thick. The surface of each $\mathrm{Si}$ block is polished to $1 \AA \mathrm{rms}$ roughness and $\lambda / 4$ at $6380 \AA .^{14}$

\section{THE BEAMLINE}

Although the beamline is used for other research, the features essential for radiation biology are illustrated in Fig. 6. Flux from the synchrotron is not deviated by any optical elements in front of the monochromator, however horizontal and vertical slits are used to define the beam entering the multilayer chamber. This is quite useful since it reduces the amount of stray light that could be transmitted by the mono-

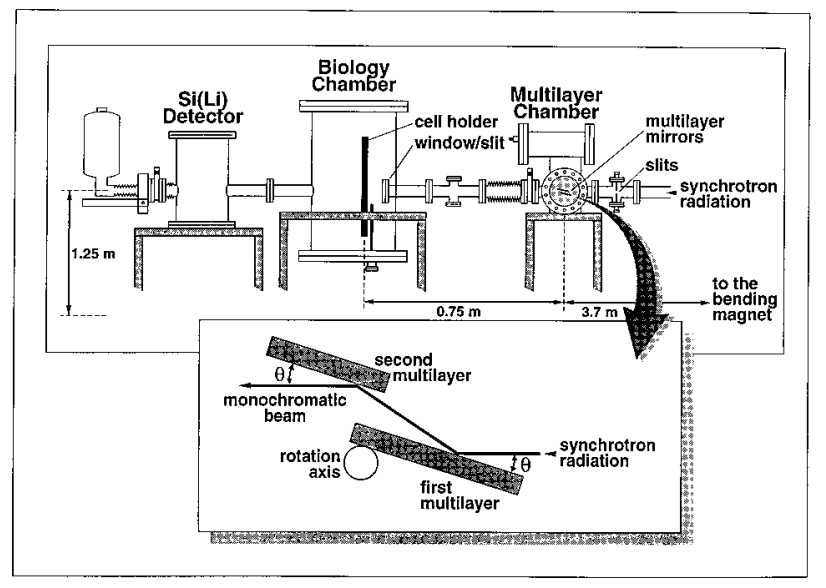

FIG. 6. Multilayer beamline configuration. chromator. The monochromator chamber is UHV compatible since it is open to the storage ring. The biology chamber is separated from the monochromator by a fixed slit vacuum window. Although the biology chamber can be pumped down to $10^{-4} \mathrm{~Pa}$, irradiations are performed at atmospheric pressure and a reliable vacuum window is essential. The $\mathrm{Si}(\mathrm{Li})$ detector after the biology chamber serves to calibrate the output from the monochromator. It should be noted that flux from the monochromator can pass through the biology and $\mathrm{Si}(\mathrm{Li})$ chambers to experimental stations positioned after the $\mathrm{Si}(\mathrm{Li})$ chamber.

The monochromator is a simple double-mirror design. Mirrors are mounted in parallel on a UHV-compatible rotary feedthrough. Each mirror is $30 \mathrm{~mm}$ wide by $60 \mathrm{~mm}$ long, with a separation distance of $10 \mathrm{~mm}$. The angular acceptance of the monochromator is $\sim 6.7 \mathrm{mrad}$. The output energy is selected by rotating the mirror assembly to change the angle of incidence. The first mirror is mounted $5 \mathrm{~mm}$ above the axis of rotation so that when rotated, it drops down into the center of beam. In this way, the full surface of the mirror may be used at grazing incidence. The angular range of the monochromator is from $5^{\circ}$ to $60^{\circ}$. For angles less than $5^{\circ}$, the reflected beam misses part of the second mirror, and for angles of incidence greater than $60^{\circ}$ the beam is blocked by the back of the first mirror. In the present version, the second mirror is not translated, therefore the output beam moves up or down as the angle of incidence is changed. The motion of the output beam limits the ability to scan the energy continuously in that realignment of downstream apparatus is required. Realignment is necessary because of the $1.6 \mathrm{~mm} \times 25$ $\mathrm{mm}$ exit slit after the monochromator. However, most of our experiments are done at a fixed energy for several weeks. It is a simple matter to realign the biology chamber downstream from the monochromator when a new energy is selected. Future upgrades of the monochromator will include the ability to translate the second mirror and scan the output energy continuously.

A fundamental limitation of any multilayer monochromator is specularly reflected light having wavelengths for which the incident angle is less than the critical angle. The output beam from the monochromator includes wavelengths that satisfy the Bragg condition as well as the longwavelength components specularly reflected from the mirror surface. The wavelengths in the specular component depend strongly on the multilayer materials and the angle of incidence of the input beam, and can be minimized to some extent by the choice of layer spacing. In all cases, visible light is strongly reflected. For applications that are sensitive to these specular components, the output beam of the monochromator must be filtered. We employ several different filtering schemes. For irradiations below the $\mathrm{C} K$ edge, a 1.5$\mu \mathrm{m}$ Mylar film is used. The Mylar is transparent between $100 \mathrm{eV}$ and the $\mathrm{C} K$ edge. With our choice of layer spacing, the specular component is mostly below $100 \mathrm{eV}$ and attenuated by the mylar. For irradiations above $600 \mathrm{eV}$, a 7.5$\mu \mathrm{m}$-thick Be filter is used. The Be filter does not pass visible light and has good transmission above $700 \mathrm{eV}$. Between the $\mathrm{C} K$ edge and $600 \mathrm{eV}$, any filter severely attenuates the 


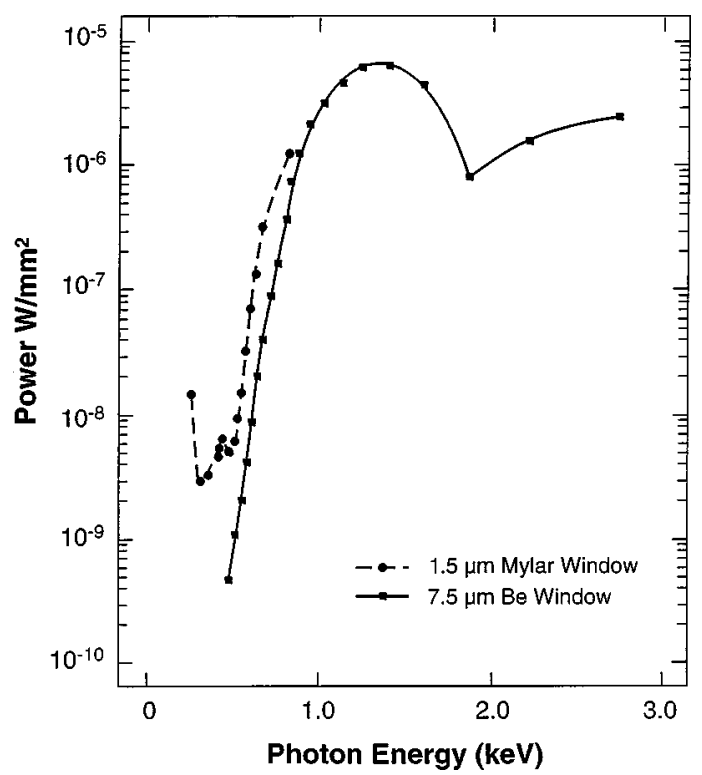

FIG. 7. Power output as a function of photon energy of the multilayer monochromator for two different filters over a $1.6 \mathrm{~mm} \times 25.0 \mathrm{~mm}$ slit: (a) $1.5-\mu \mathrm{m}$ Mylar filter; (b) $7.5-\mu \mathrm{m}$ Be filter. The line is drawn to guide the eye.

beam. Figure 7 plots output power density values, in W/ $\mathrm{mm}^{2}$, of the double-mirror monochromator measured for 1.5$\mu \mathrm{m}$ Mylar and 7.5- $\mu \mathrm{m}$ Be filters covering a $1.6 \mathrm{~mm} \times 25$ $\mathrm{mm}$ slit. The filter serves a dual role as vacuum window since it separates the biology chamber from the monochromator. The biology chamber is usually at atmospheric pressure during a cell irradiation while the monochromator is at UHV. The filter/window is made by bonding the material over a $1.6 \mathrm{~mm} \times 25 \mathrm{~mm}$ slit formed in a flange that is mounted in the biology chamber. The filter is held in place with Vacseal, and the outer edge is coated with Torrseal epoxy to provide strength and a vacuum tight seal. Be windows prepared in this manner have lasted up to two years without degradation, while the Mylar windows are only used once due to degradation from radiation damage. The storage ring vacuum is protected from window failure by a series of gate valves interlocked to the ion gauge in the monochromator chamber. We find that the Mylar windows fail slowly compared to the reaction time of the valves, and after failure the pressure in the monochromator has never risen above $\mu \mathrm{Pa}$.

Because of the presence of undesirable specular components and higher-order Bragg reflections, it is essential that the monochromator output be measured for spectral purity. For this energy range, the output beam can be monitored with a $\mathrm{Si}(\mathrm{Li})$ detector. The $\mathrm{Si}(\mathrm{Li})$ detector is regularly calibrated against the $\mathrm{O}, \mathrm{N}$ and $\mathrm{F} K$ edges to assure accuracy. Figure 8 shows the monochromator output at $273 \mathrm{eV}$ with a $1.5-\mu \mathrm{m}$ aluminized-Mylar filter. Note that there is no second-order Bragg reflection because the $\mathrm{W}$ and $\mathrm{C}$ layers are of equal thickness, and that the third-order Bragg reflection at $820 \mathrm{eV}$ is reduced by 100 compared to the first order at $273 \mathrm{eV}$. For cell survival experiments $1 \%$ third-order contamination was deemed acceptable. Measurements of the

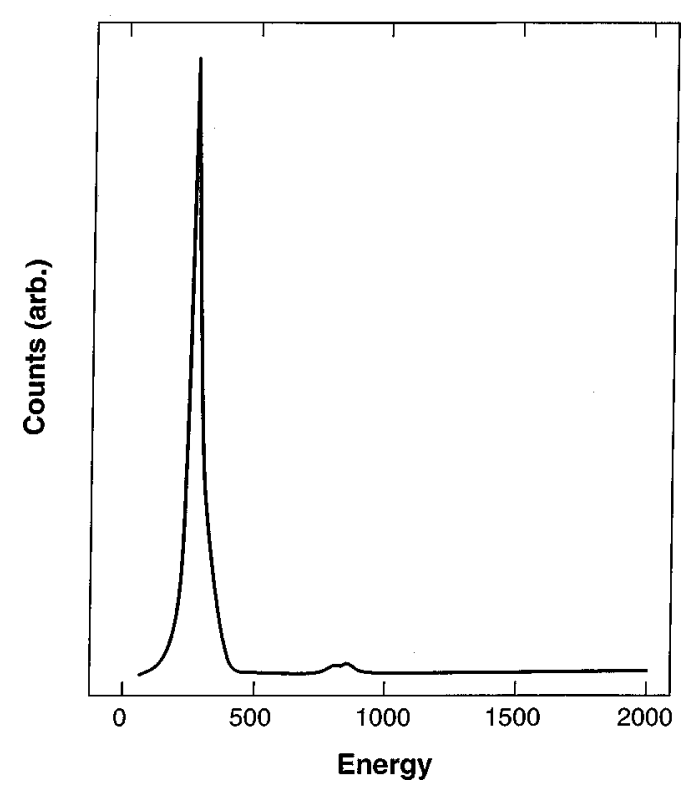

FIG. 8. Output spectrum with the monochromator set for $273 \mathrm{eV}$. The measurement was done using a $\mathrm{Si}(\mathrm{Li})$ detector which has an energy resolution less than the monochromator. The small bump near $900 \mathrm{eV}$ is the third-order reflection.

spectral content above $700 \mathrm{eV}$ yield even better results, since the output of the synchrotron for the third order is dropping rapidly in this energy range.

Our interests are to study cell survival for the isoattenuation energy pair of 273 and $860 \mathrm{eV}$ and also to study partial cellular volume irradiation using microfabricated irradiation masks with $1340-\mathrm{eV}$ photons. Mammalian cells are grown in monolayers on a 3- or 8- $\mu$ m-thick Mylar film epoxy bonded to a 25-mm-diam stainless steel cell holder. The cell holders are filled with culture medium and sealed on the opposite end with parafilm to prevent leakage of the culture medium. Up to 15 cell holders are mounted in a scanning frame that provides $x-y$ computer controlled lateral motion perpendicular to the $\mathrm{x}$-ray beam. ${ }^{15}$ In this way, the dose to each cell holder may be varied. As the cell holders must be maintained at atmospheric pressure, $\mathrm{x}$-ray attenuation is a severe problem at $273 \mathrm{eV}$ since $\mathrm{N}_{2}, \mathrm{O}_{2}$ and $\mathrm{H}_{2} \mathrm{O}$ absorb heavily at this photon energy. The distance from the exit slit to the Mylar film on the cell holders is $\sim 3 \mathrm{~mm}$, and it was decided to fill this volume with $\mathrm{H}_{2}$. Safety concerns make it impractical to fill the entire biology chamber. To limit the volume, a plastic bag is placed over the scanning frame and entry flange to the biology chamber, and $\mathrm{H}_{2}$ flows continuously into the bag. The bag inflates and moves easily with the scanning frame during the irradiation. It is desirable that the bag leak slightly so that air may be purged, and the leaking $\mathrm{H}_{2}$ is diluted with $\mathrm{N}_{2}$ that flows into the biology chamber. The mixture of $\mathrm{H}_{2}$ and $\mathrm{N}_{2}$ is continuously purged through an exhaust line so that an explosive mixture is never attained in the biology chamber. Even with $\mathrm{H}_{2}$ in the irradiation path the attenuation at $273 \mathrm{eV}$ is still significant, and places severe demands on the tolerances of the cell holders, since the length of the cell holder determines the distance from the exit slit to the cell. 


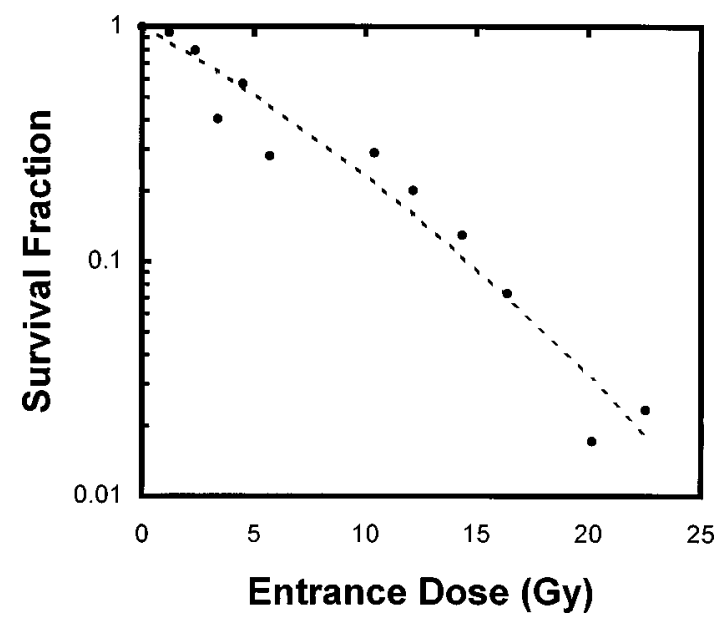

FIG. 9. Preliminary radiation survival curve. Monolayers of $10 \mathrm{~T} \frac{1}{2}$ (mouse embryo) cells were irradiated with $1340-\mathrm{eV}$ x rays. Radiation doses on the $x$ axis represent the mean entrance dose at the surface of the cells and are not corrected for attenuation. Dotted line represents a fit to $S=\exp (-\alpha D$ $\left.-\beta D^{2}\right)$.

For this reason, all of the cell holders have a length variation of less than $10 \mu \mathrm{m}$.

To quantify the biological effect of irradiation, the dose to the cells must be accurately measured. Ionization chambers or thermoluminescence detectors have been used for dosimetry, however these methods are difficult to employ under present circumstances. Recently a new type of photodiode has been developed that is stable under continuous irradiation and has excellent quantum efficiency. ${ }^{16,17}$ The diode package is small and can be placed directly in a modified cell holder in the scanning frame. In this configuration, the front surface of the diode is in the same position as the cells and directly measures the x-ray energy flux at the surface of the cells. The diode operates in photovoltaic mode and yields a current that can be easily measured with an electrometer, typically in the nA range. The current in the diode $\left(I_{d}\right)$ equals the electronic charge $(e)$ time the number of electrons $\left(n_{e}\right) . n_{e}$ is related to the number of photons per second $\left(n_{p}\right)$ striking the diode by

$$
n_{e}=n_{p} E / 3.63,
$$

where $E$ is the photon energy and 3.63 is the number of electron hole pairs per $\mathrm{eV}$ produced in Si by an $\mathrm{x}$-ray photon. The flux measured by the photodiode is then

$$
n_{p}=I_{d} 3.63 / \text { Ee . }
$$

From the flux, the surface dose to the cell may be calculated. With the observed power output, the monochromator is capable of delivering a uniform surface dose (at $273 \mathrm{eV}$ ) of up to $160 \mathrm{~J} / \mathrm{kg}$ to a $25-\mathrm{mm}$-diam cell dish in a scan time of about 15 min with a storage ring current of $100 \mathrm{~mA}$. Between 300 and $600 \mathrm{eV}$ the throughput is low and the high doses required for some cell survival studies are impractical, however, there is still usable power down to $425 \mathrm{eV}$. In the energy range from 800 to $2000 \mathrm{eV}$ the beam intensity is quite high and high doses are readily attained. In fact, for photon energies above $1000 \mathrm{eV}$, the beam intensity is too high for measuring low dose effects. Such low doses are obtained by using a "special beam" with a reduced storage ring current of $2 \mathrm{~mA}$, which gives a factor of 50 reduction in the intensity.

Initial survival data indicate that the monochromator and beamline design are well suited for biological experiments. Figure 9 shows a plot of the cell survival fraction as a function of mean surface dose for a monolayer of $10 \mathrm{~T} \frac{1}{2}$ (mouse embryo) cells. In this case, the irradiating photon energy is $1340 \mathrm{eV}$. Survival data for much higher dose have also been recorded and will be reported in detail elsewhere. With the beamline and dosimetry techniques that we have developed, this method of irradiating live cells thus becomes an important tool in studying the biological effects of ultrasoft $\mathrm{x}$ rays. Furthermore, the ability to tune the monochromatic beam to the desired photon energy offers the potential for more specific experimental design.

\section{ACKNOWLEDGMENTS}

We wish to thank D. E. Savage for many useful conversations. The biological and dosimetry work and the development of the multilayer beamline at the Synchrotron Radiation Center was funded under NIH Grant No. 5-R01 CA52475-03 and UDOE Grant No. DE-F602-86ER60417. Multilayer development was funded by NSF Grant No. 9201856. This work is based upon research conducted at the Synchrotron Radiation Center, University of Wisconsin, which is supported by the NSF under Grant No. DMR9212658.

${ }^{1}$ B. Kortright, P. Plag, R. C. Percia, P. L. Cowan, D. W. Lindle, and B. Karlin, "Multilayer-coated mirrors as power filters in synchrotron radiation beamlines," Nucl. Instrum. Methods Phys. Res. A 266, 452-456 (1988).

${ }^{2}$ Yanagihara, T. Maehara, H. Nomura, M. Yamamoto, T. Namioka, and H. Kimura, "Performance of a wideband multilayer polarizer for soft x-rays,” Rev. Sci. Instrum. 63, 1516-1518 (1992).

${ }^{3}$ T. W. Barbee, Jr., P. Pianetta, R. Readelli, R. Tatchyn, and T. W. Barbee III, "Molybdenum-silicon multilayer monochromator for the extreme ultraviolet," Appl. Phys. Lett. 50, 1841-1843 (1987).

${ }^{4}$ P. Pianetta, T. W. Barbee, Jr., and R. Readelli, "Performance of layered synthetic microstructures in monochromator applications in the soft x-ray region," Nucl. Instrum. Methods Phys. Res. A 246, 352-355 (1986).

${ }^{5}$ F. Schaefers, M. Grioni, J. Wood, H. van Brug, E. J. Puik, M. Dapor, and F. Marchetti, "Application of W/Si multilayers for monochromatization of soft x-ray synchrotron radiation," Proc. SPIE 984, 23-30 (1988).

${ }^{6}$ C. Kahn Malek, T. Moreno, R. Barchewitz, R. Rivoira, and Y. Lepetre, "Performance of large multilayer mirrors for the 0.5-1.5 nm range," Rev. Sci. Instrum. 63, 4102-4107 (1992).

${ }^{7}$ M. R. Raju, S. G. Carpenter, J. J. Chmielewski, M. E. Schillaci, M. E. Wilder, J. P. Freyer, N. F. Johnson, P. L. Schor, R. J. Sebring, and D. T. Goodhead, "Radiobiology of ultrasoft x-rays. I. Cultured Hamster cells (V79),' Radiat. Res. 110, 396-412 (1987).

${ }^{8}$ M. E. Schiiaci, S. Carpenter, M. R. Raju, R. J. Sebring, M. E. Wilder, and D T. Goodhead, "Radiobiology of ultrasoft x-rays. II. Cultured mouse cells (10T1/2)," Radiat. Res. 118, 83-92 (1989).

${ }^{9}$ W. K. Waskiewicz, D. L. Windt, J. E. Bjorkholm, L. Eichner, R. R. Freeman, T. E. Jewell, W. M. Mansfield, A. A. MacDowell, L. H. Szeto, D. M. Tennant, D. L. White, and O. R. Wood, OSA Topical Meeting on Soft X-Ray Projection Lithography, Monterey, April 1991 (unpublished).

${ }^{10}$ C. Brown, R. Z. Bachrach, S. B. M. Hagstrom, N. Lien, and C. H. Pruett, "An ultrahigh vacuum monochromator for synchrotron radiation," in 
Conference on Vacuum Ultraviolet Radiation Physics, edited by E. Koch, R. Haensel, and C. Kunz (Pergamon-Vieweg, Braunschweig, 1974),p. 785 .

${ }^{11}$ D. E. Savage, J. Kleiner, N. Schimke, Y.-H. Phang, T. Jankowski, J. Jacobs, R. Kariotis, and M. G. Lagally 'Determination of roughness correlations in multilayer films for x-ray mirrors,' J. Appl. Phys. 69, 1411-1424 (1991).

${ }^{12}$ M. G. Lagally, Kinetics of Ordering and Growth at Surfaces (Plenum, New York, 1990).

${ }^{13}$ Y. H. Phang, R. Kariotis, D. E. Savage, and M. G. Lagally "Diffraction from multilayer films with partially correlated interfacial roughness," J. Appl. Phys. 72, 4627 (1992).
${ }^{14}$ Innovative Optical Technologies, 700 Weaver Park Road, Longmont, CO 50501.

${ }^{15}$ C. M. Meger, D. W. Pearson, P. M. DeLuca, Jr., F. Cerrina, and M. G. Gould, "Beamline and irradiation chamber for dosimetry and biology studies using synchrotron radiation,' Rev. Sci. Instrum. 60, 2235-2238 (1989).

${ }^{16}$ L. R. Canfield, J. Kerner, and R. Korde, "Stability and quantum efficiency performance of silicon photodiode detectors in the far ultraviolet," Appl. Opt. 28, 3940-3943 (1989).

${ }^{17}$ AXUV-100 photodiode from International Radiation Detectors (IRD), 2545 W237th St. Suite I, Torrance, CA 90505. 